\title{
ALS User Meeting Features 10th Anniversary Celebration
}

The Advanced Light Source (ALS) saw first light on October 5 1993, not quite to the day but close to 10 years before the 2003 ALS Users' Association meeting, held October 6-8. No one among the 289 registered attendees sang "Happy birthday to you," but there were slides with birthday cakes in the presentations by ALS Director Daniel Chemla and U.S. Department of Energy (DOE) Associate Director of Basic Energy Sciences Patricia Dehmer.

Fittingly, the proceedings included retrospectives from two of the key players in the early history of the ALS, David Attwood and Jay Marx, and a display of photographs chronicling its construction and commissioning. Science highlights, highlights from young researchers, and posters constituted the meat of the program on Monday and Tuesday morning, while workshops took over Tuesday afternoon and all day Wednesday. Generous support from equipment vendors underwrote refreshments during the receptions at the end of the first two days.

Monday morning included many of the staples of user meetings around the world with greetings from the host management, perspectives from representatives of funding agencies, DOE in this case, and facility status reports. To start it off, Berkeley Lab Director Charles Shank welcomed attendees with a spirited endorsement of the plans for an ALS performance upgrade that would keep the facility at the forefront of the field for the next two decades.

Chemla presented a report on past accomplishments and future plans, including a look at the kinds of science to come. Featured prominently in his description, the proposed upgrade would increase the ALS brightness without reducing the beam lifetime by means of quasi-continuous injection (top-off), increased beam current (up to $1 \mathrm{amp}$ ), smaller beam size, advanced insertion devices (elliptically polarizing, superconducting, and narrow-gap in-vacuum), and application-specific beamlines optimized for one class of experiments. Replacing the old 5-meter-long undulators with two shorter devices in the same straight section would also increase the number of undulator beamlines. A phased, four-year project would avoid lengthy shutdowns and minimize disruption to users.

After a casting a brief glimpse at the history of third-generation of synchrotron facilities in the U.S., Dehmer turned her attention to the 20-year roadmap then under development for facilities development within DOE's Office of Science, whose broad portfolio includes Basic Energy Sciences and five other offices responsible for research from elementary particles to biology. She noted that the highest recommendation of the Basic Energy Sciences Advisory Committee (BESAC) was for "Light Source Facilities Upgrades.” The roadmap subsequently announced by DOE on November 11, 2003 includes several such synchrotron light source upgrades spaced over many years.

The remainder of the morning was devoted to a Town Meeting. ALS Deputy for Operations Ben Feinberg emphasized safety, including a new effort to clean up the increasingly busy experiment hall floor, and summarized plans for a new user building, to 
be completed by early 2008. Accelerator Physics Group Leader David Robin reviewed the ingredients in the accelerator portion of the ALS upgrade, primarily quasi-continuous injection (top-off) at $1.9 \mathrm{GeV}$ and a higher beam current. ALS Deputy for Science Neville Smith noted that determining the complement of advanced insertion devices and application-specific beamlines for the upgrade could not be done without user input. ALS User Services Group Leader Gary Krebs briefly reviewed the hopeful status of a proposed on-site user housing facility. Finally, outgoing Users' Executive Committee chair Jennifer Doudna (University of California, Berkeley) encouraged users to participate in a letter-writing campaign to influence pending legislation that could increase funding for synchrotron facilities and science.

Science highlights presented on Monday afternoon and Tuesday morning were intended to illustrate some of the frontier areas of user research at the ALS. The rapidly developing area of ultrafast x-ray studies was represented by Ernie Glover (Berkeley Lab), who spoke on "Picosecond Metal-Insulator Transitions in an Expanding Metallic Fluid: Kinetics of Particle Formation.” Two presentations by Wanli Yang (Stanford University and Berkeley Lab) on "Angle-Resolved Photoemission Reveals $\mathrm{C}_{60}$ Electronic Structure" and Kai Starke (Freie University Berlin) on "Fermi Surface Nesting in Terbium Metal" were in the core research area of condensed-matter physics.

Moving away from hard matter, Terrill Cool's (Cornell University) review of "Studies of Flame Chemistry Using Synchrotron Radiation" illustrated a new direction in chemical dynamics at the ALS. A presentation on "What Prevents Complete Mixing of Alcohol and Water" by Jinghua Guo (Berkeley Lab) explored the growing area of electronic spectroscopy of water-based systems. Carolyn Larabell (University of California, San Francisco, and Berkeley Lab) showed how to combine cryocooling and tomography in her talk on "Imaging Cells with X Rays.” And Sheryl Tsai (University of California, Irvine) represented the increasingly large protein-crystallography activity at the ALS with her talk "The Architecture and Specificity of Acyl-CoA Carboxylase ß Subunit."

Young researchers are the future of the field and are featured in ALS user meetings. This year, the frontier topics of ultrafast imaging and the use of coherence to study magnetic nanostructures were well represented. Sug-Bong Choe (Berkeley Lab) spoke on "Time and Spatially Resolved Microscopy on Magnetization Dynamics Using X-PEEM," and Karine Chesnel (Berkeley Lab) reported on "Magnetic Speckle from Nanostructures." Strong electron correlation effects were featured in talks by Norman Manella (University of California, Davis) on "Direct Observation of High-Temperature Polaronic Behavior in Colossal Magnetoresistive Manganites" and by Byron Freelon (Berkeley Lab) on "Electron Correlation and Charge Transfer in $\left(\mathrm{Ba}_{0.9} \mathrm{Nd}_{0.1}\right) \mathrm{CuO}_{2+\mathrm{d}} /\left(\mathrm{CaCuO}_{2}\right)_{2}$," Wendel Caldwell (Berkeley Lab) described the use of microdiffraction in his presentation "Shear at Twin Domain Boundaries in $\mathrm{YBa}_{2} \mathrm{Cu}_{3} \mathrm{O}_{7-\mathrm{x} .}$,"

To celebrate the 10th anniverary of ALS operation, the user meeting program featured looks back at the origin of the ALS and at its construction. David Attwood (University of California, Berkeley, and Berkeley Lab) recounted the realization of "The Impossible Dream," as the ALS moved from a concept to an approved project. Jay Marx (Berkeley 
Lab) was the project director during ALS construction and provided his view in "The Construction of the ALS-A Personal Retrospective,"). Both of these presentations are posted on the ALS Web site at www-als.lbl.gov.

Immediately following the general session portion of the ALS users' meeting, several focused workshops were held Tuesday afternoon and all day Wednesday. Workshop titles and organizers this year were: "Applications of Variable Polarization in Soft X-Ray Microscopy and Spectroscopy,” Elke Arenholz and Tony Young (Berkeley Lab), "Inelastic X-Ray Scattering and Ultrahigh-Resolution Photoemission Studies of Strongly Correlated Systems," M. Zahid Hasan (Princeton University) and Alessandra Lanzara (University of California, Berkeley, and ALS); and "Macromolecular Crystallography," Gerry McDermott and Corie Ralston (Berkeley Lab).

In addition, as the Stanford Synchrotron Radiation Laboratory (SSRL) and the ALS now schedule their user meetings to take place the same week, a number of joint workshops are held, some of which take place at the ALS and some at SSRL. This year the title and organizers of the joint ALS-SSRL workshop held at the ALS was "Recent Advances in Synchrotron-Based Microscopy,” Greg Denbeaux, (SUNY-Albany), Katharina Luening (SSRL), Gary Mitchell (Dow Chemical), Piero Pianetta (SSRL), and David Shuh (Berkeley Lab). Titles and organizers of joint workshop held at SSRL were "Probing Mechanical Deformation and Failure via Synchrotron X Rays,” Apurva Mehta (SSRL) and Nobumichi Tamura (ALS); and "Synchrotron Techniques for Environmental Microbiology and Biogeochemistry,” John Bargar (SSRL) and David Shuh (Berkeley Lab).

Individual reports for two of the four workshops held at the ALS follow this article. Reports for workshops held at SSRL will appear with the SSRL user meeting report (see SRN 17.x, pp. xx-yy).

After a buffet dinner on Tuesday evening, user meeting co-chairs Eli Rotenberg (ALS) and Gerry McDermott (Berkeley Lab) presented awards. The student poster competition was won by Tonio Buonassisi (University of California, Berkeley) for a study of copper contaminants in polycrystalline silicon solar cell material. The Tim Renner User Services Award went to Donna Hamamoto (ALS Beamline Coordination). Xiang-Jiang Zhou (Stanford University and Berkeley Lab) took home the David A. Shirley Award for Outstanding Science for his angle-resolved photoemission studies of high-temperature superconductors. And Ruth Halbach (Klaus' widow) presented the Klaus Halbach Award for Instrumentation Development to Mark Le Gros (Berkeley Lab) for developing an automated tomography station for x-ray microscopy of biological materials.

Capping the evening's activities was a dedication of the main ALS conference room to Halbach, inventor of the permanent-magnet insertion devices that in part motivated building the ALS and other third-generation synchrotron light sources optimized for high brightness.

Art Robinson 
Advanced Light Source

Berkeley Laboratory

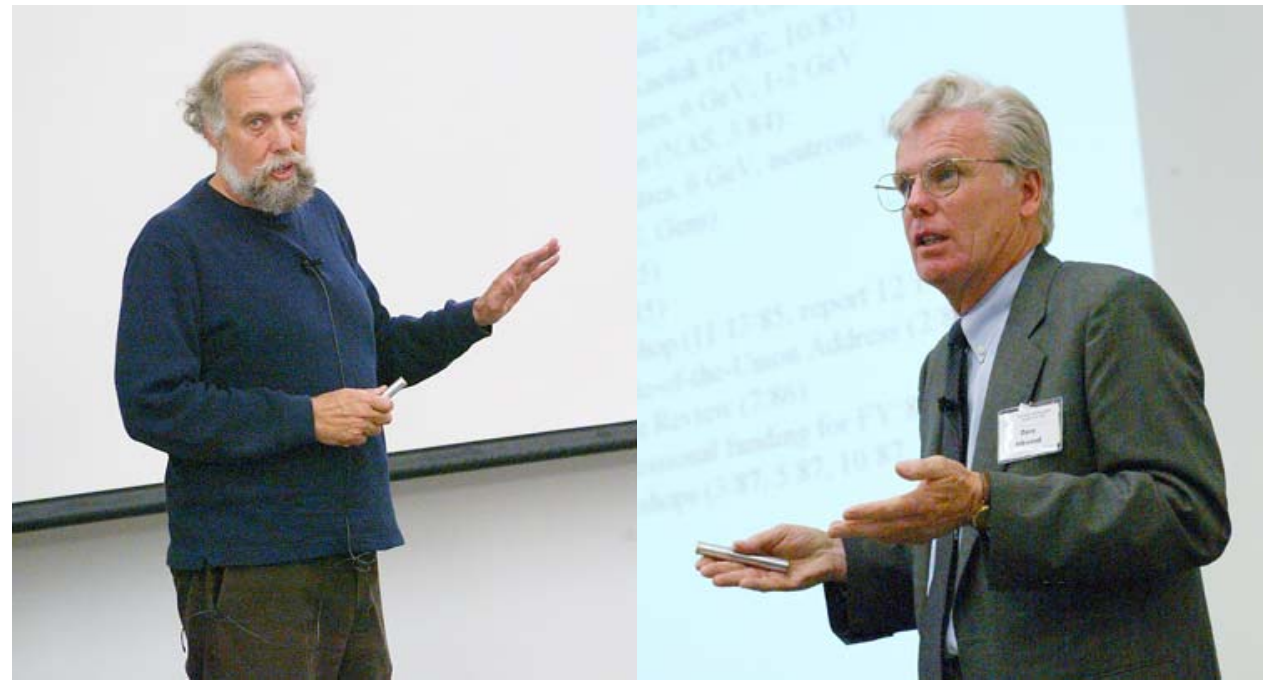

Figure 1: (left) XBD200310-00646-43.tif and (right) XBD200310-00646-37.tif. Former Project Director Jay Marx and former Scientific Director David Attwood help celebrate the ALS' tenth anniversary of operation with accounts of the early history of the facility from initial concept through construction.

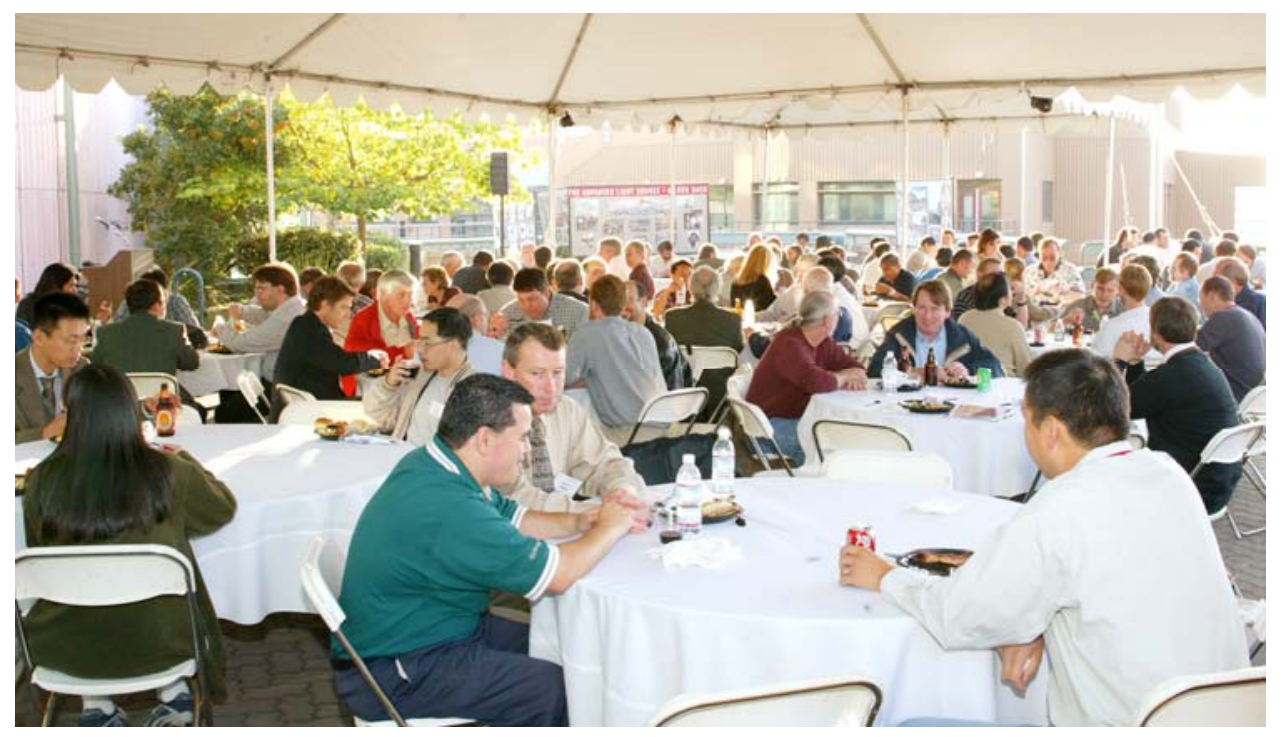

Figure 2: XBD200310-00647-37.tif

After a busy poster session in the exhibitor's tent, the traditional outdoor buffet/awards dinner took place on the ALS patio. 


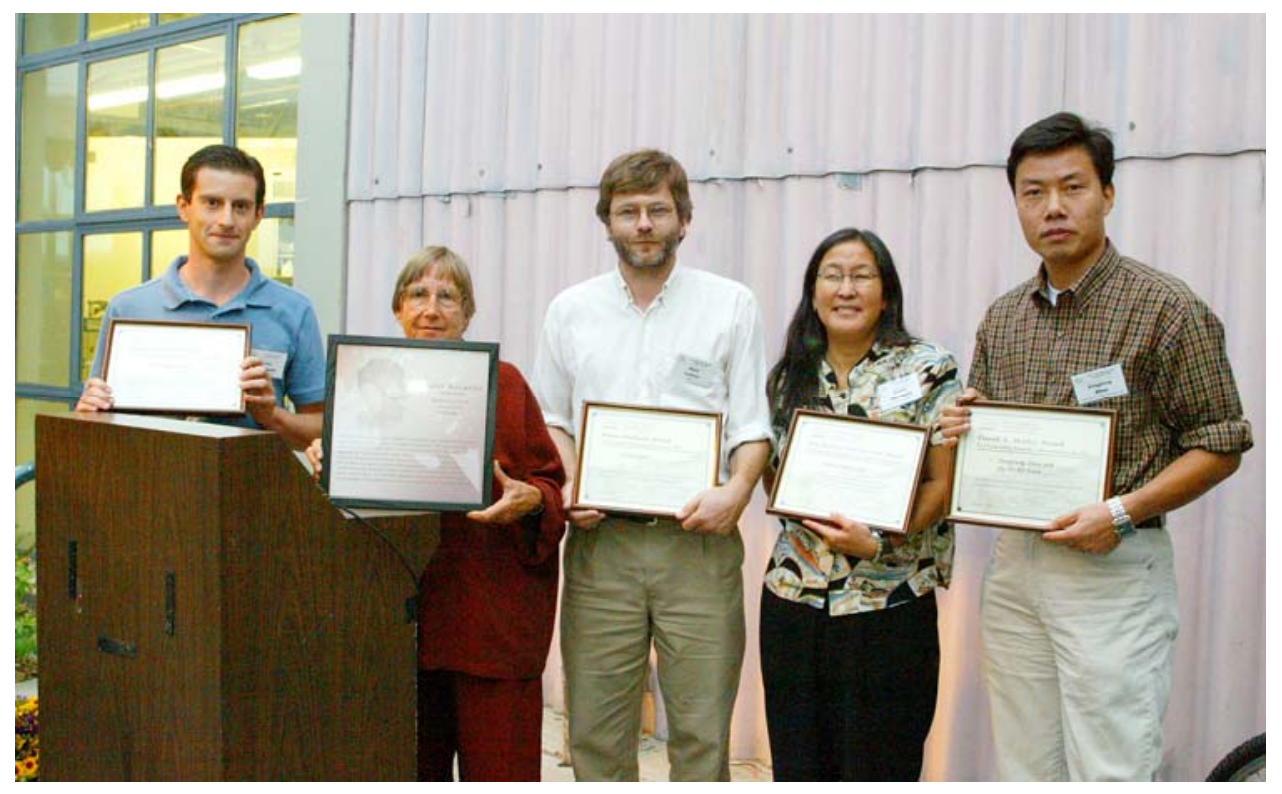

Figure 3: XBD200310-00647-90.tif

Contributions to outstanding science, instrumentation, and user support were acknowledged during the dinner. From left, Tonio Buonassisi (Student Poster Award), Ruth Halbach (holding special dedication plaque in appreciation of Klaus Halbach), Mark Le Gros (Klaus Halbach Award), Donna Hamamoto (Tim Renner User Services Award), and Xing-Jiang Zhou (David A. Shirley Award) 